\title{
Rapid Identification and Systematic Mechanism of Flavonoids from Potentilla freyniana Bornm. Based on UHPLC-Q-Exactive Orbitrap Mass Spectrometry and Network Pharmacology
}

\author{
Wei Cai $\mathbb{D}^{1,2}$ Kailin Li $\mathbb{D},{ }^{2}$ Shihan Qin, ${ }^{3}$ Pei Xiong $\mathbb{D}^{2}$, Jie Peng $\mathbb{D}^{2}$ Silin Shi $\mathbb{D}$, \\ and Zaiqi Zhang $\mathbb{D}^{1}$ \\ ${ }^{1}$ Hunan Provincial Key Laboratory of Dong Medicine, Hunan University of Medicine, Huaihua 41800, China \\ ${ }^{2}$ School of Pharmaceutical Sciences, Hunan University of Medicine, Huaihua 41800, China \\ ${ }^{3}$ School of Pharmacy, Weifang Medical University, Weifang 261053, China \\ Correspondence should be addressed to Zaiqi Zhang; qizaizhang@126.com
}

Received 11 October 2020; Revised 27 November 2020; Accepted 16 January 2021; Published 27 January 2021

Academic Editor: Richard G. Brereton

Copyright $\odot 2021$ Wei Cai et al. This is an open access article distributed under the Creative Commons Attribution License, which permits unrestricted use, distribution, and reproduction in any medium, provided the original work is properly cited.

\begin{abstract}
Potentilla freyniana Bornm. (P. freyniana), belonging to the family Rosaceae, has been used as a folk medicine in China. However, as we know, the constituents and the systematic elucidation of the mechanism were not fully investigated. Therefore, it is necessary to develop a rapid method using LC-MS and network pharmacology for the detection and identification of constituents and the systematic mechanism of $P$. freyniana. Firstly, the flavonoids were detected and identified based on ultra-high-performance liquid chromatography coupled with Quadrupole-Exactive Focus Orbitrap MS (UHPLC-Q-Exactive Orbitrap MS). After that, the potential targets of those constituents were obtained by database mining. Then, the core targets were predicted by protein-protein interaction network and network analysis. Finally, Gene Ontology (GO) and Kyoto Encyclopedia of Genes and Genomes (KEGG) pathway enrichment analysis were carried out via DAVID. This finding revealed that $P$. freyniana possessed 43 flavonoids (40 of them were first reported) with 23 core target genes, which are associated with PI3K-Akt, MAPK, TNF signaling pathway, and pathway in cancer. This study demonstrated the multicompound, multitarget, and multimechanism of P. freyniana, which are very beneficial to develop the further study and utilization of this plant including the material basis and quality control research.
\end{abstract}

\section{Introduction}

Potentilla freyniana Bornm. (P. freyniana), a genus Potentilla of the family Rosaceae, named Difengzi, is a perennial plant with branched and tufted roots widely distributed and cultivated allaround of China, especially in Hunan, Hubei, Jiangxi. Their roots have been used as a folk medicine in clearing away heat and toxic materials for treating canker, bone tuberculosis, external bleeding [1-3]. Previous investigations on P. freyniana showed the presence of different compounds including terpenes and flavonoids [4-7] and possessed a variety of activities such as anti-inflammatory, analgesic effects $[8,9]$. However, as we know, the constituents and systematic pharmacological mechanism were not fully investigated. For instance, 14 compounds, including eriodictyol, phlorizin, were separated from the roots of $P$. freyniana [7]. Therefore, it is worthwhile to establish a highly sensitive method for characterizing their chemical constituents and elucidating systematic pharmacological mechanism of P. freyniana.

The complexity of chemicals in Traditional Chinese Medicine (TCM) including P. freyniana has presented a significant challenge in the rapid identification and characterization of components. During the past decades, HPLCMS, as a new technique has been used to profile and identify the chemical in TCM due to its validity, sensitivity, and specialness [10, 11]. Especially, UHPLC-HRMS such as UHPLC-Q-Exactive Orbitrap MS, UHPLC-Q-TOF MS, and UHPLC-LTQ-Orbitrap-MS, affording a higher and faster 
separation and higher resolution of mass, was a much more powerful equipment in the identification of TCM compared to traditional HPLC-MS [12-14].

Network pharmacology is an impressive methodology for investigating the systematic pharmacological mechanism through the constructing and analyzing biological networks such as protein-protein interaction, chemical-target-pathway network, which could provide direction for the further discovery of new drug without enormous time, money, and effort [15-17].

Therefore, this current study was designed to develop a fast and effective method for the chemical characterization and systematic pharmacological mechanism of P. freyniana using UHPLC-Q-Exactive Orbitrap MS and network pharmacology. $P$. freyniana possessed 43 flavonoids (40 of them was first reported) with 23 core target genes, which are associated with PI3K-Akt, MAPK, TNF signaling pathway, and pathway in cancer. This study demonstrated the multicompound, multitarget, and multimechanism of $P$. freyniana, which are very beneficial for the further study and utilization of this plant including the material basis and quality control research.

\section{Materials and Methods}

2.1. Chemicals and Materials. The chemical reference standards isoquercitrin, luteolin, naringenin, and kaempferol were provided by Chengdu Herbpurify biotechnology CO., LTD (Chengdu, China); Phlorizin, Phloretin, and Trilobatin were purchased from Sichuan Wei Keqi Biotechnology Co., Ltd (Sichuan, China); eriodictyol and hyperoside were provided by Chengdu Push biotechnology CO., LTD (Chengdu, China); quercetin and apigenin were provided by Chengdu Alfa biotechnology CO., LTD (Chengdu, China); baicalein and wogonin were obtained from Chengdu Desite biotechnology CO., LTD (Chengdu, China). The purities of all chemical reference standards were above $98 \%$ by HPLC-DAD.

Acetonitrile and methanol of chromatography grade were provided by MERCK (Darmstadt, Germany); The ultrapure water was produced by a milli-Q water purification system (Millipore, Milford, MA, United States); formic acid of LC-MS grade and all other reagents of analytical grade were purchased from Aladdin Industrial Corporation.

$P$. freyniana was collected from Tong-Dao country of Huaihua (109.86 longitude, 26.03 latitude), Hunan province and were identified by Professor Wei Cai (Hunan Provincial Key Laboratory of Dong Medicine, Hunan University of Medicine). The voucher specimen was deposited at School of Pharmaceutical Sciences, Hunan University of Medicine.

2.2. Sample Preparation. A total of $10 \mathrm{~g}$ powdered root of P. freyniana was ultrasonically extracted with $50 \mathrm{~mL}$ of $70 \%$ aqueous methanol for $1 \mathrm{~h}$, and then the extracted solution was filtered for further UHPLC Q-Exactive Focus Orbitrap MS analysis.

The reference standards including hyperoside, isoquercitrin, phlorizin, eriodictyol, trilobatin, quercetin, luteolin, naringenin, apigenin, phloretin, kaempferol, baicalein, and wogonin were weighed and dissolved in methanol to obtain the reference solution with the final concentrations of $10.2,9.8,10.0,9.8,9.9,10.5,10.2,10.8,9.3$, $10.1,10.6,9,4$, and $10.5 \mathrm{ug} / \mathrm{mL}$, respectively, and then these solutions were stored in $-4^{\circ} \mathrm{C}$ before analysis.

2.3. Instruments and Conditions. Chromatography analysis was performed on an Ultimate 3000 focused system (Dionex, Sunnyvale, CA, USA) consisting of an online vacuum degasser, a binary pump, and an autosampler. The sample separation was carried out on the Hypersil GOLD C18 column $(100 \times 2.1 \mathrm{~mm}, 1.9 \mu \mathrm{m})$ at $40^{\circ} \mathrm{C}$. The mobile phase consisted of acetonitrile as solvent $\mathrm{A}$ and water with $0.1 \%$ formic acid as solvent $\mathrm{B}$ using the following gradient elution of $5-15 \% \mathrm{~A}$ at $0-2 \mathrm{~min}$; isocratic $15 \% \mathrm{~A}$ at $2-5 \mathrm{~min} ; 15-18 \%$ $\mathrm{A}$ at $5-10 \mathrm{~min} ; 18-50 \% \mathrm{~A}$ at $10-15 \mathrm{~min} ; 50-100 \% \mathrm{~A}$ at $15-16 \mathrm{~min}$; $100-5 \% \mathrm{~A}$ at $16-17 \mathrm{~min}$; isocratic $5 \% \mathrm{~A}$ at $17-20 \mathrm{~min}$. The flow rate was $0.3 \mathrm{~mL} / \mathrm{min}$.

All LC-MS ${ }^{\mathrm{n}}$ analyses were performed on the Q-Exactive Focus Orbitrap MS connected to the UHPLC system via a heated electrospray ionization source (Thermo Electron, Bremen, Germany). The optimized tune operating parameters in negative ion mode were listed as follows: sheath gas and auxiliary gas flow rate of 30 and 10 arbitrary, respectively; the capillary and auxiliary gas heater temperatures of $320^{\circ} \mathrm{C}$ and $350^{\circ} \mathrm{C}$, respectively; spray voltage of $3.0 \mathrm{kV}$; RF lens of 50 ; High-resolution MS analysis was performed at full scan $\mathrm{MS}^{1}$ with the mass range of $\mathrm{m} / \mathrm{z} 100-1000$ at a resolution of 35000 and targeted $\mathrm{MS}^{2}$ at a resolution of 17500 triggered by parallel reaction monitoring mode; nitrogen was set as sheath, auxiliary, and collision gas; the isolation widow was $2 \mathrm{amu}$, and the normalized collision energy (NCE) was $30 \%$.

2.4. Data Processing and Analysis. All high-resolution MS data were acquired and processed using the Xcalibur version (2.0 software, Thermo Fisher Scientific, San Jose, CA, USA). The compounds were detected by the Compound Discover version 3 using the metabolism workflow templates by the expected compounds predicted method [18]. The detailed parameters of the workflow template were set as follows: the minimum peak intensity was set as 10000; the maximum element counts were $\mathrm{C} 30 \mathrm{H} 60 \mathrm{O} 20$; the mass tolerance of MS and $\mathrm{MS}^{2}$ was within 5 and $10 \mathrm{ppm}$, respectively; baicalein and phloretin were set as the carbon skeleton; reduction, oxidation was set as Phase I transformation; glucoside conjugation, glucuronide conjugation, pentoside conjugation, methylation was set as Phase II transformation.

2.5. Target Identification of Flavonoids. TCMSP database is a free and online database for potential target identification of small molecules, especially TCM. The target genes were converted to the official gene symbol by STRING (https:// string-db.org) or Uniport (https://www.uniprot.org).

2.6. Protein-Protein Interaction Network. STRING was a free tool, which can construct the PPI network by uploading the potential targets. The species was set as "Homo sapiens" with 
a confidence score $>0.4$. The network analysis was performed at Cytoscape to obtain the core targets.

\subsection{GeneMANIA Analysis. GeneMANIA (http://} genemania.org) is an online free and friendly tool for investigating gene function and gene interaction. The species was set as "Homo sapiens."

2.8. GO and Pathway Analysis. The GO and KEGG pathway analysis was performed on the DAVID (https://david. ncifcrf.gov, Version 6.8). The specific species in the list and background was set as "Homo sapiens." The entire compounds, targets, and pathway network were visualized by Cytoscape.

\section{Results and Discussion}

3.1. Analytical Strategy. In order to identify flavonoids fully, an analytical strategy based on UHPLC Q-Exactive Focus Orbitrap MS was established in this study. First, the sample was prepared and injected into the UHPLC Q-Exactive Focus Orbitrap MS to gain the full scan high-resolution MS data. Then, those data were processed using Compound Discover software with metabolism workflow to predict and detect the molecule of flavonoids. Third, the $\mathrm{MS}^{2}$ of the predicted molecule were acquired using UHPLC Q-Exactive Focus Orbitrap MS by parallel reaction monitoring mode. Finally, the compounds were identified based on the full scan MS, MS $^{2}$ data, retention time, and bibliography.

3.2. Identification of Flavonoids. The total content of flavonoids was measured by $\mathrm{NaNO}_{2}-\mathrm{Al}\left(\mathrm{NO}_{3}\right)_{3}-\mathrm{NaOH}$ spectrophotometric colorimetry [19]. The calibration curve obtained by the rutin standard of absorbance concentrations $(\mathrm{mg} / \mathrm{mL})$ using five dilutions was $y=7.15 x-0.001$, with the corresponding determination coefficient at 0.9999 . Finally, the content of flavonoids is $32.17 \pm 0.26 \%$. A total of 43 constituents were unanimously and tentatively characterized based on UHPLC Q-Exactive Focus Orbitrap MS combined with the expected compounds predicted method. 40 excluded eriodictyol, phloretin, and hyperoside were reported from $P$. freyniana for the first time. The detailed information of those compounds is listed in Table 1 . The high-resolution extracted ion chromatography is shown in Figure 1.

Peaks $15,17,27,33,35-43$ were unanimously identified as hyperoside, isoquercitrin, phlorizin, eriodictyol, trilobatin, quercetin, luteolin, naringenin, apigenin, phloretin, kaempferol, baicalein, and wogonin, respectively, by comparing the retention time, high-resolution mass measurement, and $\mathrm{MS}^{2}$ spectrum with those reference standards.

Peak 13 was eluted at $6.57 \mathrm{~min}$ and possessed the deprotonated ion $[\mathrm{M}-\mathrm{H}]^{-}$at $\mathrm{m} / \mathrm{z} 303.0507(-0.99 \mathrm{ppm}$, $\mathrm{C}_{15} \mathrm{H}_{11} \mathrm{O}_{7}$ ). The fragment ions at $\mathrm{m} / \mathrm{z} 125.0234$ (-8.14 ppm, $\left.\mathrm{C}_{6} \mathrm{H}_{5} \mathrm{O}_{3}\right)$ and $285.0407\left(0.84 \mathrm{ppm}, \mathrm{C}_{15} \mathrm{H}_{9} \mathrm{O}_{6}\right)$ were detected in the $\mathrm{MS}^{2}$ spectrum, which is consistent with the MS data of taxifolin in bibliography [20]. Thus, peak 13 was tentatively identified as taxifolin. Peaks $1-3$, and 8 possessed the deprotonated ion $[\mathrm{M}-\mathrm{H}]^{-}$at $\mathrm{m} / \mathrm{z} \quad 465.1039$ (0.21 ppm, $\left.\mathrm{C}_{21} \mathrm{H}_{21} \mathrm{O}_{12}\right), \mathrm{m} / \mathrm{z} 465.1033$ (-1.08 ppm, $\left.\mathrm{C}_{21} \mathrm{H}_{21} \mathrm{O}_{12}\right), \mathrm{m} / \mathrm{z}$ 465.1042 (0.86 ppm, $\left.\mathrm{C}_{21} \mathrm{H}_{21} \mathrm{O}_{12}\right)$, and $\mathrm{m} / \mathrm{z} \quad 465.1042$ (0.86 ppm, $\left.\mathrm{C}_{21} \mathrm{H}_{21} \mathrm{O}_{12}\right)$, respectively, $162 \mathrm{Da}\left(\mathrm{C}_{6} \mathrm{H}_{10} \mathrm{O}_{5}\right.$, glucose moiety) more than that of taxifolin (peak 13). The fragmentation ions at $\mathrm{m} / \mathrm{z} 285.041\left(\mathrm{C}_{15} \mathrm{H}_{9} \mathrm{O}_{6}\right)$, 125.023 $\left(\mathrm{C}_{6} \mathrm{H}_{5} \mathrm{O}_{3}\right), 303.051\left(\mathrm{C}_{15} \mathrm{H}_{11} \mathrm{O}_{7}\right)$ in the $\mathrm{MS}^{2}$ spectrum were matched to those attributed to taxifolin. Therefore, Peaks $1-3$, and 8 were tentatively characterized as taxifolinglucoside.

Peaks 4 and 26 were eluted at 4.13 and $11.18 \mathrm{~min}$, respectively. All of them showed the same deprotonated ion $[\mathrm{M}-\mathrm{H}]^{-}$at $\mathrm{m} / \mathrm{z} 463.088\left(\mathrm{C}_{21} \mathrm{H}_{19} \mathrm{O}_{12}\right), 176.032 \mathrm{Da}\left(\mathrm{C}_{6} \mathrm{H}_{8} \mathrm{O}_{6}\right.$, glucuronide moiety) more than that of eriodictyol, suggesting they are eriodictyol-glucuronide, which were further identified by the presence of fragmentation ion at $\mathrm{m} / \mathrm{z}$ $287.056\left(\mathrm{C}_{15} \mathrm{H}_{11} \mathrm{O}_{6}\right)$. In a similar way, peaks 9,28 , and 30 were tentatively identified as taxifolin-glucuronide, quercetin-glucuronide, and quercetin-glucuronide, respectively.

Peaks 5, 7, 10, 16, 19, 22, and 34 were eluted at 4.19, 4.56, $5.88,6.90,7.99,9.61$, and $13.57 \mathrm{~min}$, with the same deprotonated ion $[\mathrm{M}-\mathrm{H}]-$ at $\mathrm{m} / \mathrm{z} 449.109\left(\mathrm{C}_{21} \mathrm{H}_{21} \mathrm{O}_{11}\right)$. Peaks 19 and 34 possessed the fragment ions at $\mathrm{m} / \mathrm{z} 167.034\left(\mathrm{C}_{8} \mathrm{H}_{7} \mathrm{O}_{4}\right)$ and $\mathrm{m} / \mathrm{z} 123.044\left(\mathrm{C}_{7} \mathrm{H}_{7} \mathrm{O}_{2}\right)$, which are the diagnosis fragmentation ions of phloretin, suggesting they were phloretin derivatives. Thus, Peaks 19 and 34 were tentatively inferred as phloretin-glucuronide. Peaks 5, 7, 10, 16, and 22 yielded the same fragmentation ion at $\mathrm{m} / \mathrm{z} 287.056\left(\mathrm{C}_{15} \mathrm{H}_{11} \mathrm{O}_{6}\right)$, suggesting they were eriodictyol derivatives. The ion at $\mathrm{m} / \mathrm{z}$ 287.056 was yielded by the neutral loss of $162.053\left(\mathrm{C}_{6} \mathrm{H}_{10} \mathrm{O}_{5}\right.$, glucose moiety), suggesting the presence of glucose moiety. Therefore, they were tentatively characterized as eriodictyolglucoside.

Peak 6 with the deprotonated ion $[\mathrm{M}-\mathrm{H}]-$ at $\mathrm{m} / \mathrm{z}$ 593.1535 (3.88 ppm, $\mathrm{C}_{27} \mathrm{H}_{29} \mathrm{O}_{15}$ ) was eluted at $4.41 \mathrm{~min}$. It yielded fragment ions at $\mathrm{m} / \mathrm{z} \quad 353.0667 \quad(0.07 \mathrm{ppm}$, $\left.\mathrm{C}_{19} \mathrm{H}_{13} \mathrm{O}_{7}\right), \quad 383.0774 \quad\left(0.42 \mathrm{ppm}, \quad \mathrm{C}_{20} \mathrm{H}_{15} \mathrm{O}_{8}\right)$, 473.1092(0.56 ppm, $\left.\mathrm{C}_{23} \mathrm{H}_{21} \mathrm{O}_{11}\right)$, and $413.0874(-0.98 \mathrm{ppm}$, $\mathrm{C}_{21} \mathrm{H}_{17} \mathrm{O}_{9}$ ), resulting from the loss of $\mathrm{C}_{4} \mathrm{H}_{8} \mathrm{O}_{4}+\mathrm{C}_{4} \mathrm{H}_{8} \mathrm{O}_{4}$, $\mathrm{C}_{4} \mathrm{H}_{8} \mathrm{O}_{4}+\mathrm{C}_{3} \mathrm{H}_{6} \mathrm{O}_{3}, \mathrm{C}_{4} \mathrm{H}_{8} \mathrm{O}_{4}$, and $\mathrm{C}_{3} \mathrm{H}_{6} \mathrm{O}_{3}+\mathrm{C}_{3} \mathrm{H}_{6} \mathrm{O}_{3}$, respectively, suggesting the presence of two carbon-glucoside. According to the published paper [21,22], peak 6 was tentatively identified as Vicenin II. In a similar way, peak 18 was tentatively identified as Phloretin-C-diglucoside.

Peaks 11, 14, 24, and 31 generated the same quasimolecular ion $[\mathrm{M}-\mathrm{H}]-$ at $\mathrm{m} / \mathrm{z} 433.114\left(\mathrm{C}_{21} \mathrm{H}_{21} \mathrm{O}_{10}\right), 162$ $\mathrm{Da}\left(\mathrm{C}_{6} \mathrm{H}_{10} \mathrm{O}_{5}\right.$, glucose moiety) more than that of naringenin (peak 38), suggesting they were naringenin-glucoside, which were further confirmed by the presence of $\mathrm{m} / \mathrm{z} 271.061$ and 151.003 in $\mathrm{MS}^{2}$ spectrum.

Peak 12 eluted at $6.22 \mathrm{~min}$ and showed a pseudomolecular ion at $\mathrm{m} / \mathrm{z} 625.1408\left(0.00 \mathrm{ppm}, \mathrm{C}_{27} \mathrm{H}_{29} \mathrm{O}_{17}\right), 176.032$ $\mathrm{Da}\left(\mathrm{C}_{6} \mathrm{H}_{8} \mathrm{O}_{6}\right.$, glucuronide moiety) more than that of eriodictyol-glucoside, suggesting it is eriodictyol-glucosideglucuronide, which was confirmed by the presence of the base peak at $\mathrm{m} / \mathrm{z} 287.0558$ (eriodictyol).

Peaks 20, 21, 25, and 29 presented the same deprotonated ion $[\mathrm{M}-\mathrm{H}]-$ at $\mathrm{m} / \mathrm{z} 431.099\left(\mathrm{C}_{21} \mathrm{H}_{19} \mathrm{O}_{10}\right)$ and generated the 
TABLE 1: The retention time and mass spectrometric data of flavone in Potentilla freyniana Bornm.

\begin{tabular}{|c|c|c|c|c|c|c|c|}
\hline Peak & $t_{R}$ & $\begin{array}{l}\text { Theoretical } \\
\text { mass, } \mathrm{m} / \mathrm{z}\end{array}$ & $\begin{array}{l}\text { Experimental } \\
\text { mass, } \mathrm{m} / \mathrm{z}\end{array}$ & $\begin{array}{l}\text { Error } \\
(\mathrm{ppm})\end{array}$ & Formula & MS/MS fragment & Identification \\
\hline 1 & 3.59 & 465.1038 & 465.1039 & 0.21 & $\mathrm{C}_{21} \mathrm{H}_{22} \mathrm{O}_{12}$ & $\begin{array}{c}\mathrm{MS}^{2}[465]: 285.0406(100), 125.0233(72), \\
275.0563(45), 177.0185(29), 303.0512(20), \\
151.0032(17)\end{array}$ & Taxifolin-glucoside \\
\hline 2 & 3.72 & 465.1038 & 465.1033 & -1.08 & $\mathrm{C}_{21} \mathrm{H}_{22} \mathrm{O}_{12}$ & $\begin{array}{c}\mathrm{MS}^{2}[465]: 285.0405(100), 125.0233(38) \\
273.0043(28), 177.0185(19), 303.0511(15)\end{array}$ & Taxifolin-glucoside \\
\hline 3 & 3.88 & 465.1038 & 465.1042 & 0.86 & $\mathrm{C}_{21} \mathrm{H}_{22} \mathrm{O}_{12}$ & $\begin{array}{l}\mathrm{MS}^{2}[465]: 285.0405(100), 125.0233(36) \\
177.0184(18), 275.0566(12), 303.0512(10)\end{array}$ & Taxifolin-glucoside \\
\hline 4 & 4.13 & 463.0882 & 463.0881 & -0.22 & $\mathrm{C}_{21} \mathrm{H}_{20} \mathrm{O}_{12}$ & $\begin{array}{c}\mathrm{MS}^{2}[463]: 287.0562(100), 259.0612(78) \\
125.0232(32)\end{array}$ & $\begin{array}{l}\text { Eriodictyol- } \\
\text { glucuronide }\end{array}$ \\
\hline 5 & 4.19 & 449.1089 & 449.1093 & 0.89 & $\mathrm{C}_{21} \mathrm{H}_{22} \mathrm{O}_{11}$ & $\begin{array}{c}\mathrm{MS}^{2}[449]: 259.0611(100), 287.0561(33) \\
178.9979(15), 125.0231(13)\end{array}$ & $\begin{array}{l}\text { Eriodictyol- } \\
\text { glucoside }\end{array}$ \\
\hline 6 & 4.41 & 593.1512 & 593.1535 & 3.88 & $\mathrm{C}_{27} \mathrm{H}_{30} \mathrm{O}_{15}$ & $\begin{array}{l}\mathrm{MS}^{2}[593]: 353.0667(100), 383.0774(54) \\
473.1092(35), 125.0234(25), 413.0874(6)\end{array}$ & Vicenin II \\
\hline 7 & 4.56 & 449.1089 & 449.1092 & 0.67 & $\mathrm{C}_{21} \mathrm{H}_{22} \mathrm{O}_{11}$ & $\begin{array}{l}\mathrm{MS}^{2}[449]: 259.0612(100), 269.0455(76) \\
287.0563(44), 125.0233(38), 178.9977(22)\end{array}$ & $\begin{array}{l}\text { Eriodictyol- } \\
\text { glucoside }\end{array}$ \\
\hline 8 & 5.61 & 465.1038 & 465.1042 & 0.86 & $\mathrm{C}_{21} \mathrm{H}_{22} \mathrm{O}_{12}$ & $\begin{array}{c}\mathrm{MS}^{2}[465]: 125.0233(100), 285.0405(62) \\
259.0610(48), 275.0566(32), 303.0512(18)\end{array}$ & Taxifolin-glucoside \\
\hline 9 & 5.75 & 479.0831 & 479.0834 & 0.63 & $\mathrm{C}_{21} \mathrm{H}_{20} \mathrm{O}_{13}$ & $\begin{array}{c}\mathrm{MS}^{2}[479]: 285.0405(100), 125.0233(41) \\
303.0512(18), \\
\text { 259.0613(14) }\end{array}$ & $\begin{array}{l}\text { Taxifolin- } \\
\text { glucuronide }\end{array}$ \\
\hline 10 & 5.88 & 449.1089 & 449.1092 & 0.67 & $\mathrm{C}_{21} \mathrm{H}_{22} \mathrm{O}_{11}$ & $\begin{aligned} \mathrm{MS}^{2}[449]: & 269.0456(100), 151.0026(69) \\
178.9979(39), & 125.0231(13), 259.0612(12), \\
& 287.0562(10)\end{aligned}$ & $\begin{array}{l}\text { Eriodictyol- } \\
\text { glucoside }\end{array}$ \\
\hline 11 & 6.12 & 433.1140 & 433.1142 & 0.46 & $\mathrm{C}_{21} \mathrm{H}_{22} \mathrm{O}_{10}$ & $\begin{array}{c}\mathrm{MS}^{2}[433]: 271.0614(100), 151.0027(73) \\
119.0491(18), 125.0229(8)\end{array}$ & $\begin{array}{l}\text { Naringenin- } \\
\text { glucoside }\end{array}$ \\
\hline 12 & 6.22 & 625.1408 & 625.1408 & 0.00 & $\mathrm{C}_{27} \mathrm{H}_{30} \mathrm{O}_{17}$ & $\begin{array}{c}\mathrm{MS}^{2}[625]: 287.0558(100), 113.0231(40) \\
151.0030(38)\end{array}$ & $\begin{array}{l}\text { Eriodictyol- } \\
\text { glucoside- } \\
\text { glucuronide }\end{array}$ \\
\hline 13 & 6.57 & 303.0510 & 303.0507 & -0.99 & $\mathrm{C}_{15} \mathrm{H}_{12} \mathrm{O}_{7}$ & $\mathrm{MS}^{2}[303]: 125.0234(100), 285.0407(38)$ & Taxifolin \\
\hline 14 & 6.59 & 433.1140 & 433.1141 & 0.23 & $\mathrm{C}_{21} \mathrm{H}_{22} \mathrm{O}_{10}$ & $\begin{array}{c}\mathrm{MS}^{2}[433]: 271.0613(100), 151.0027(72) \\
119.0491(16)\end{array}$ & $\begin{array}{l}\text { Naringenin- } \\
\text { glucoside }\end{array}$ \\
\hline $15^{*}$ & 6.85 & 463.0882 & 463.0886 & 0.86 & $\mathrm{C}_{21} \mathrm{H}_{20} \mathrm{O}_{12}$ & MS2[463]: 300.0272(100), 301.0350(70) & Hyperoside \\
\hline 16 & 6.90 & 449.1089 & 449.1094 & 1.11 & $\mathrm{C}_{21} \mathrm{H}_{22} \mathrm{O}_{11}$ & $\begin{array}{c}\mathrm{MS}^{2}[449]: 151.0028(100), 287.0562(73) \\
135.0442(32)\end{array}$ & $\begin{array}{l}\text { Eriodictyol- } \\
\text { glucoside }\end{array}$ \\
\hline $17^{*}$ & 7.18 & 463.0882 & 463.0886 & 0.86 & $\mathrm{C}_{21} \mathrm{H}_{20} \mathrm{O}_{12}$ & $\begin{array}{c}\text { MS2 [463]: } 300.0279(100), 301.0358(50) \\
151.0025(8), 178.9976(7)\end{array}$ & Isoquercitrin \\
\hline 18 & 7.75 & 597.1825 & 597.1832 & 1.17 & $\mathrm{C}_{27} \mathrm{H}_{34} \mathrm{O}_{15}$ & $\begin{array}{l}\mathrm{MS}^{2}[597]: 357.0983(100), 387.1087(85) \\
315.0882(22), 417.1165(21), 358.1008(15)\end{array}$ & $\begin{array}{l}\text { Phloretin-C- } \\
\text { diglucoside }\end{array}$ \\
\hline 19 & 7.99 & 449.1089 & 449.1085 & -0.89 & $\mathrm{C}_{21} \mathrm{H}_{22} \mathrm{O}_{11}$ & $\begin{array}{c}\mathrm{MS}^{2}[449]: \text { 167.0340(69), 137.0233(50), } \\
123.0441(12)\end{array}$ & $\begin{array}{l}\text { Phloretin- } \\
\text { glucuronide }\end{array}$ \\
\hline 20 & 8.15 & 431.0984 & 431.0992 & 1.86 & $\mathrm{C}_{21} \mathrm{H}_{20} \mathrm{O}_{10}$ & $\operatorname{MS}^{2}[431]: 269.0445(100)$ & Baicalein-glucoside \\
\hline 21 & 9.60 & 431.0984 & 431.0988 & 0.93 & $\mathrm{C}_{21} \mathrm{H}_{20} \mathrm{O}_{10}$ & $\operatorname{MS}^{2}[431]: 269.0443(100)$ & Baicalein-glucoside \\
\hline 22 & 9.61 & 449.1089 & 449.1086 & -0.67 & $\mathrm{C}_{21} \mathrm{H}_{22} \mathrm{O}_{11}$ & $\begin{array}{c}\mathrm{MS}^{2}[449]: \\
151.0026(100), 287.0563(65) \\
135.0442(22)\end{array}$ & $\begin{array}{l}\text { Eriodictyol- } \\
\text { glucoside }\end{array}$ \\
\hline 23 & 9.68 & 565.1563 & 565.1568 & 0.88 & $\mathrm{C}_{26} \mathrm{H}_{30} \mathrm{O}_{14}$ & $\mathrm{MS}^{2}[565]: 271.0614(100), 151.0026(48)$ & $\begin{array}{l}\text { Naringenin- } \\
\text { pentoside- } \\
\text { glucoside }\end{array}$ \\
\hline 24 & 9.82 & 433.1140 & 433.1142 & 0.46 & $\mathrm{C}_{21} \mathrm{H}_{22} \mathrm{O}_{10}$ & $\begin{array}{c}\mathrm{MS}^{2}[433]: 271.0615(100), 151.0028(41) \\
119.0491(14)\end{array}$ & $\begin{array}{l}\text { Naringenin- } \\
\text { glucoside }\end{array}$ \\
\hline 25 & 10.38 & 431.0984 & 431.0992 & 1.86 & $\mathrm{C}_{21} \mathrm{H}_{20} \mathrm{O}_{10}$ & $\mathrm{MS}^{2}[431]:$ 268.0369(100), 269.0444(47) & $\begin{array}{l}\text { Apigenin-7- } \\
\text { glucoside }\end{array}$ \\
\hline 26 & 11.18 & 463.0882 & 463.0886 & 0.86 & $\mathrm{C}_{21} \mathrm{H}_{20} \mathrm{O}_{12}$ & $\begin{array}{c}\mathrm{MS}^{2}[463]: 151.0027(100), 113.0231(66) \\
287.0562(52), \\
161.0234(40), 337.0569(39), \\
135.0439(23)\end{array}$ & $\begin{array}{l}\text { Eriodictyol- } \\
\text { glucuronide }\end{array}$ \\
\hline $27^{*}$ & 12.16 & 435.1297 & 435.1300 & 0.69 & $\mathrm{C}_{21} \mathrm{H}_{24} \mathrm{O}_{10}$ & $\begin{array}{c}\mathrm{MS}^{2}[435]: 273.0769(100), 167.0340(69) \\
125.00232(9), 179.0341(6)\end{array}$ & Phlorizin \\
\hline 28 & 12.36 & 477.0674 & 477.0678 & 0.84 & $\mathrm{C}_{21} \mathrm{H}_{18} \mathrm{O}_{13}$ & $\begin{array}{c}\mathrm{MS}^{2}[477]: 301.0353(100), 151.0029(11) \\
178.9983(9)\end{array}$ & $\begin{array}{l}\text { Quercetin- } \\
\text { glucuronide }\end{array}$ \\
\hline
\end{tabular}


TABLE 1: Continued.

\begin{tabular}{|c|c|c|c|c|c|c|c|}
\hline Peak & $t_{R}$ & $\begin{array}{l}\text { Theoretical } \\
\text { mass, } \mathrm{m} / \mathrm{z}\end{array}$ & $\begin{array}{l}\text { Experimental } \\
\text { mass, } \mathrm{m} / \mathrm{z}\end{array}$ & $\begin{array}{l}\text { Error } \\
(\mathrm{ppm})\end{array}$ & Formula & MS/MS fragment & Identification \\
\hline 29 & 12.51 & 431.0984 & 431.0986 & 0.46 & $\mathrm{C}_{21} \mathrm{H}_{20} \mathrm{O}_{10}$ & $\begin{array}{c}\mathrm{MS}^{2}[431]: 268.0371(100), 269.0441(18), \\
239.0337(9)\end{array}$ & $\begin{array}{l}\text { Apigenin- } 4^{\prime}- \\
\text { glucoside }\end{array}$ \\
\hline 30 & 12.62 & 477.0674 & 477.0678 & 0.84 & $\mathrm{C}_{21} \mathrm{H}_{18} \mathrm{O}_{13}$ & $\mathrm{MS}^{2}[477]:$ 301.0354(100), $178.9983(12)$ & $\begin{array}{l}\text { Quercetin- } \\
\text { glucuronide }\end{array}$ \\
\hline 31 & 13.00 & 433.1140 & 433.1144 & 0.92 & $\mathrm{C}_{21} \mathrm{H}_{22} \mathrm{O}_{10}$ & $\mathrm{MS}^{2}[433]: 271.0612(100), 151.0027(46)$ & $\begin{array}{l}\text { Naringenin- } \\
\text { glucoside }\end{array}$ \\
\hline 32 & 13.39 & 567.1719 & 567.1730 & 1.94 & $\mathrm{C}_{26} \mathrm{H}_{32} \mathrm{O}_{14}$ & $\begin{array}{c}\mathrm{MS}^{2}[567]: 273.0771(100), 167.0340(42) \\
125.0020(22)\end{array}$ & $\begin{array}{l}\text { Phloretin- } \\
\text { pentoside- } \\
\text { glucoside }\end{array}$ \\
\hline $33^{*}$ & 13.45 & 287.0561 & 287.0564 & 1.05 & $\mathrm{C}_{15} \mathrm{H}_{12} \mathrm{O}_{6}$ & $\begin{array}{c}\text { MS2[287]: } 151.0027(100), 135.0441(69), \\
107.0125(10)\end{array}$ & Eriodictyol \\
\hline 34 & 13.57 & 449.1089 & 449.1085 & -0.89 & $\mathrm{C}_{21} \mathrm{H}_{22} \mathrm{O}_{11}$ & $\begin{array}{c}\mathrm{MS}^{2}[449]: 137.0233(100), 167.0340(89) \\
123.0440(8)\end{array}$ & $\begin{array}{l}\text { Phloretin- } \\
\text { glucuronide }\end{array}$ \\
\hline $35^{*}$ & 13.67 & 435.1297 & 435.1303 & 1.38 & $\mathrm{C}_{21} \mathrm{H}_{24} \mathrm{O}_{10}$ & $\mathrm{MS}^{2}[435]:$ 273.0767(100), 167.0339(52) & Trilobatin \\
\hline $36^{*}$ & 13.86 & 301.0354 & 301.0356 & 0.66 & $\mathrm{C}_{15} \mathrm{H}_{10} \mathrm{O}_{7}$ & $\begin{array}{c}\operatorname{MS}^{2}[301]: 151.0027(100), 178.9978(66) \\
121.0284(21), 107.0125(8)\end{array}$ & Quercetin \\
\hline $37^{*}$ & 13.95 & 285.0405 & 285.0407 & 0.70 & $\mathrm{C}_{15} \mathrm{H}_{10} \mathrm{O}_{6}$ & $\begin{array}{c}\mathrm{MS}^{2}[285]: 285.0404(100), 151.0033(12) \\
133.0290(11), 175.0395(8)\end{array}$ & Luteolin \\
\hline $38^{*}$ & 14.79 & 271.0611 & 271.0614 & 1.11 & $\mathrm{C}_{15} \mathrm{H}_{12} \mathrm{O}_{5}$ & $\begin{array}{c}\mathrm{MS}^{2}[271]: 151.0027(100), 119.0499(36) \\
177.0184(14), 93.0333(14), 107.0126(9)\end{array}$ & Naringenin \\
\hline $39^{*}$ & 14.95 & 269.0455 & 269.0454 & -0.37 & $\mathrm{C}_{15} \mathrm{H}_{10} \mathrm{O}_{5}$ & $\begin{array}{c}\mathrm{MS}^{2}[269]: 269.0455(100), 117.0344(8) \\
149.0239(7), 151.0035(7)\end{array}$ & Apigenin \\
\hline $40^{*}$ & 14.99 & 273.0768 & 273.0771 & 1.10 & $\mathrm{C}_{15} \mathrm{H}_{14} \mathrm{O}_{5}$ & $\begin{array}{c}\operatorname{MS}^{2}[273]: 167.0341(100), 123.0438(18) \\
119.0491(12), 125.0231(8)\end{array}$ & Phloretin \\
\hline $41^{*}$ & 15.07 & 285.0405 & 285.0405 & 0.00 & $\mathrm{C}_{15} \mathrm{H}_{10} \mathrm{O}_{6}$ & $\mathrm{MS}^{2}[285]: 285.0403(100), 151.0030(5)$ & Kaempferol \\
\hline $42^{*}$ & 15.49 & 269.0455 & 269.0453 & -0.74 & $\mathrm{C}_{15} \mathrm{H}_{10} \mathrm{O}_{5}$ & $\begin{array}{c}\mathrm{MS}^{2}[269]: 269.0453(100), 241.0504(9), \\
251.0348(8), 223.0399(7)\end{array}$ & Baicalein \\
\hline $43^{*}$ & 16.71 & 283.0612 & 283.0613 & 0.35 & $\mathrm{C}_{16} \mathrm{H}_{12} \mathrm{O}_{5}$ & $\operatorname{MS}^{2}[283]: 268.0376(100), 163.0035(7)$ & Wogonin \\
\hline
\end{tabular}

${ }^{*}$ Identified by comparing with reference standards.

same fragment ions at $\mathrm{m} / \mathrm{z} 269.044\left(\mathrm{C}_{15} \mathrm{H}_{9} \mathrm{O}_{5}\right)$ by loss of the glucose moiety $\left(\mathrm{C}_{6} \mathrm{H}_{10} \mathrm{O}_{5}\right)$, which suggested the presence of glucose moiety. The base peak at $\mathrm{m} / \mathrm{z} 268.037\left[\mathrm{Y}_{0}-\mathrm{H}\right]$ ions in the $\mathrm{MS}^{2}$ spectrum of peaks 25 and 29 was a characteristic of apigenin aglycone. According to the published paper [23, 24], they were tentatively inferred as apigenin-7-glucoside and apigenin- $4^{\prime}$-glucoside, respectively. Meantime, peaks 20 and 21 were tentatively characterized as baicalein-glucoside.

Peak 32 was detected at $13.39 \mathrm{~min}$. It presented a pseudomolecular ion at $\mathrm{m} / \mathrm{z} 567.1730\left(1.94 \mathrm{ppm}, \mathrm{C}_{26} \mathrm{H}_{32} \mathrm{O}_{14}\right)$ and exhibited the $\mathrm{MS}^{2}$ fragmentation ions at $\mathrm{m} / \mathrm{z} 273.0771$ (1.10 ppm, $\mathrm{C}_{15} \mathrm{H}_{13} \mathrm{O}_{5}$ ), resulting from the loss of glucose moiety and pentoside moiety (294.096). Thus, peak 32 was tentatively characterized as phloretin-pentoside-glucoside. In a similar way, peak 23 was tentatively identified as naringenin-pentoside-glucoside.

3.3. Target Identification of Flavonoids. 212 putative targets of flavonoids were obtained from the TCMSP database. A visual compounds-targets network with 224 nodes and 440 edges was built by Cytoscape Version 3.7.2 (Figure S1). Compounds quercetin, apigenin, kaempferol, luteolin, and wogonin are the top 5 compounds with a maximum degree and betweenness in the compound-targets network. The detailed information of putative targets linked to compounds was provided in Supplementary Table S1.
3.4. Protein-Protein Interaction Network. In order to find the key targets of flavonoids, a total of 212 putative targets were imported into the STRING to obtain the protein-protein interaction (PPI) data. The PPI network with 206 nodes and 3980 edges was established by Cytoscape (Figure S2). A total of 23 targets, including AKT1, INS, TP53, IL6, HSP90AA1, EGFR, VEGFA, JUN, EGF, CASP3, MAPK1, ESR1, ERBB2, PTGS2, MYC, MAPK8, MMP9, FN1, FOS, PPARG, CXCL8, CYCS, and CCND1, were selected as the core targets for GO and KEGG pathway analysis by setting the parameters as follows: the degree $\geq 50$; betweenness centrality $\geq 0.01$; closeness centrality $\geq 0.6$.

3.5. GeneMANIA Analysis. Among the 23 key target genes and their interacting genes, it was found that $42.75 \%$ had coexpression characteristics, $41.10 \%$ displayed physical interactions characteristic. Other characteristics, including pathway, genetic interactions, colocalization, and shared protein domains, are displayed in Figure 2.

3.6. GO and Pathway Analysis. In order to further study the 23 core target genes, GO and KEGG pathway analysis were performed by DAVID. GO term enrichment analysis results were divided into the biological process (BP, 23/23), cell compound (CC, 23/23), and molecular function (MF, 23/ 23). A total of $158 \mathrm{BP}, 16 \mathrm{CC}$, and $33 \mathrm{MF}$ has a $p$-value less 


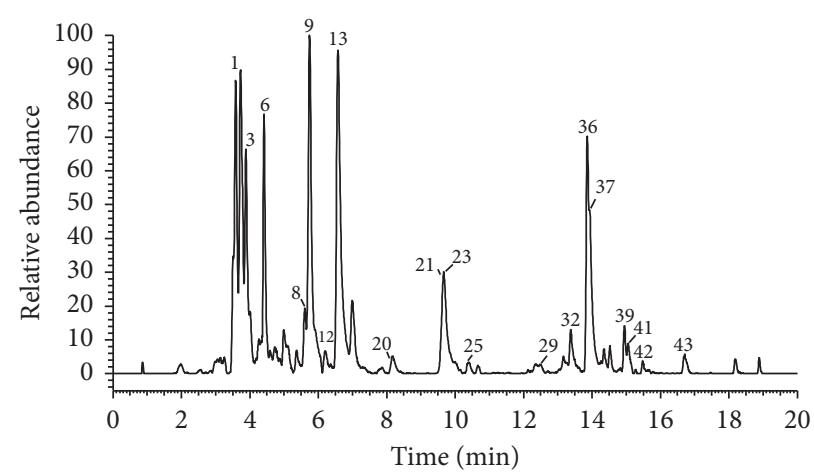

(a)

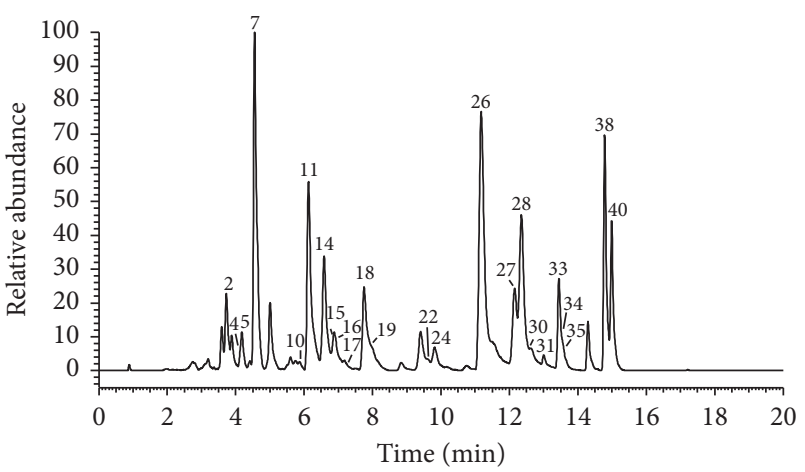

(b)

FIGURE 1: The high-resolution extracted ion chromatogram (HREIC) in $5 \mathrm{ppm}$ for the multiple compounds in Potentilla freyniana Bornm. (a) $\mathrm{m} / \mathrm{z} 269.0455,283.0612,285.0405,301.0354,303.0510,431.0984,465.1038,479.0831,565.1563,567.1719,593.1512,625.1408 ;$ (b) $\mathrm{m} / \mathrm{z}$ 271.0611, 273.0768, 287.0561, 433.1140, 435.1297, 449.1089, 463.0882, 465.1038, 477.0674, 597.1825.

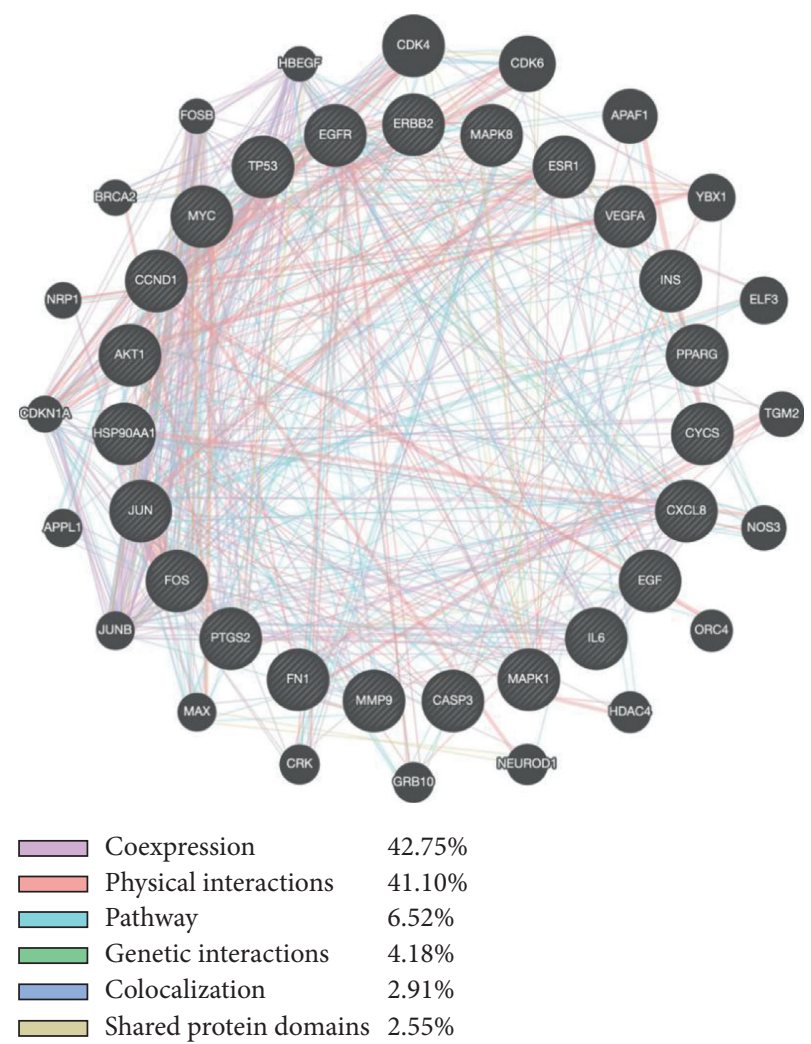

FIGURe 2: Protein network of core target genes by GeneMANIA. Black nodes represent target proteins, and connecting colors indicate different correlations.

than 0.05 (Table S2). In GO term enrichment analysis, the BP might be related to positive regulation of transcription from RNA polymerase II promoter (10/23), response to drug (9/23), negative regulation of apoptotic process (9/ 23), positive regulation of transcription, DNA-templated $(9 / 23)$, signal transduction $(9 / 23)$, positive regulation of gene expression (8/23), and positive regulation of cell proliferation (8/23), and so on. The top 4 of CC are nucleus $(16 / 23)$, nucleoplasm (12/23), cytosol (12/23), and cytoplasm (12/23). The MF are protein binding on $100 \%$, identical protein binding on $56.5 \%$, enzyme binding on $39.1 \%$, and transcription factor binding on $34.8 \%$. The top 10 enriched terms in BP, CC, and MF are displayed in Figure 3. In addition, 83 KEGG pathways (Table S3) were enriched as $p$-value less than 0.05 . The result showed that the pathway was mainly related to the signaling pathway including PI3K-Akt (12/23), MAPK (10/23), TNF (9/23), ErbB (8/23), HIF-1 (8/23), Estrogen (8/23), FoxO (8/23), and cancer in the pathway. The top 20 KEGG pathways are shown in Figure 4. 


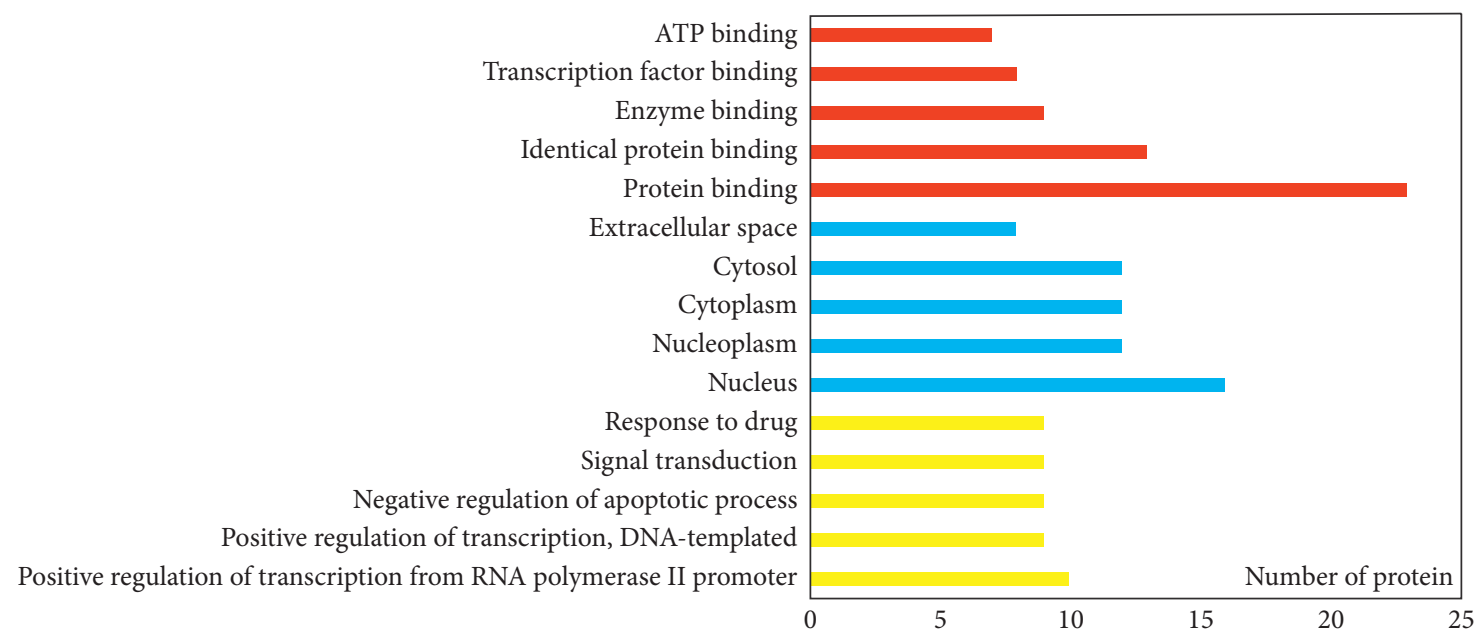

FIGURE 3: GO analysis by DAVID: red represents the biological process, yellow represents the cellular component, green represents the molecular function.

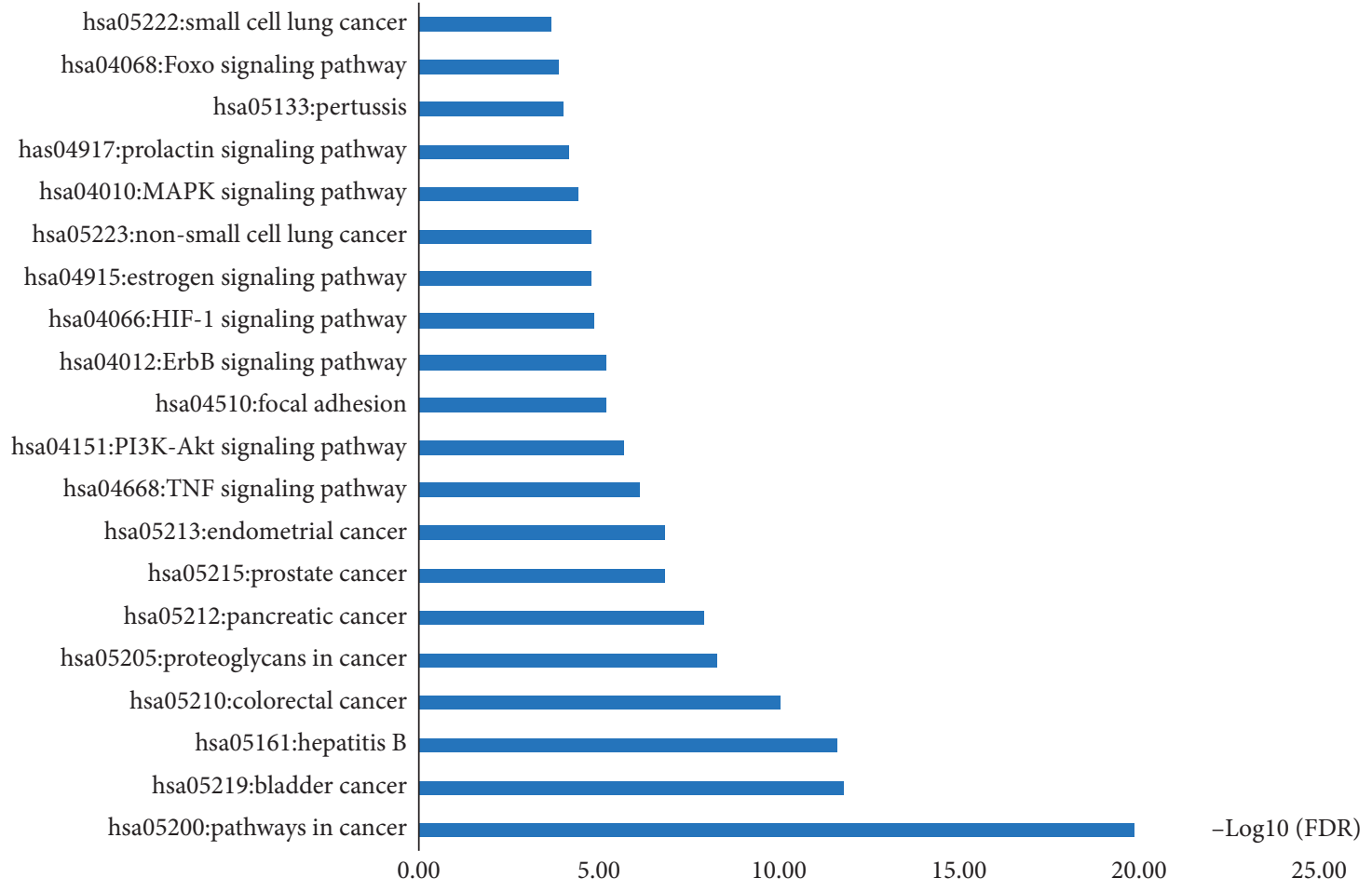

FIGURE 4: KEGG pathway analysis.

3.7. Network Analysis. Based on the target and KEGG pathway analysis, the entire compounds, targets, and pathway network were established by Cytoscape. The network with 122 nodes and 595 edges is shown in Figure 5. The red diamond, green ellipse, and blue triangle represent compounds, genes, and pathways, respectively. 


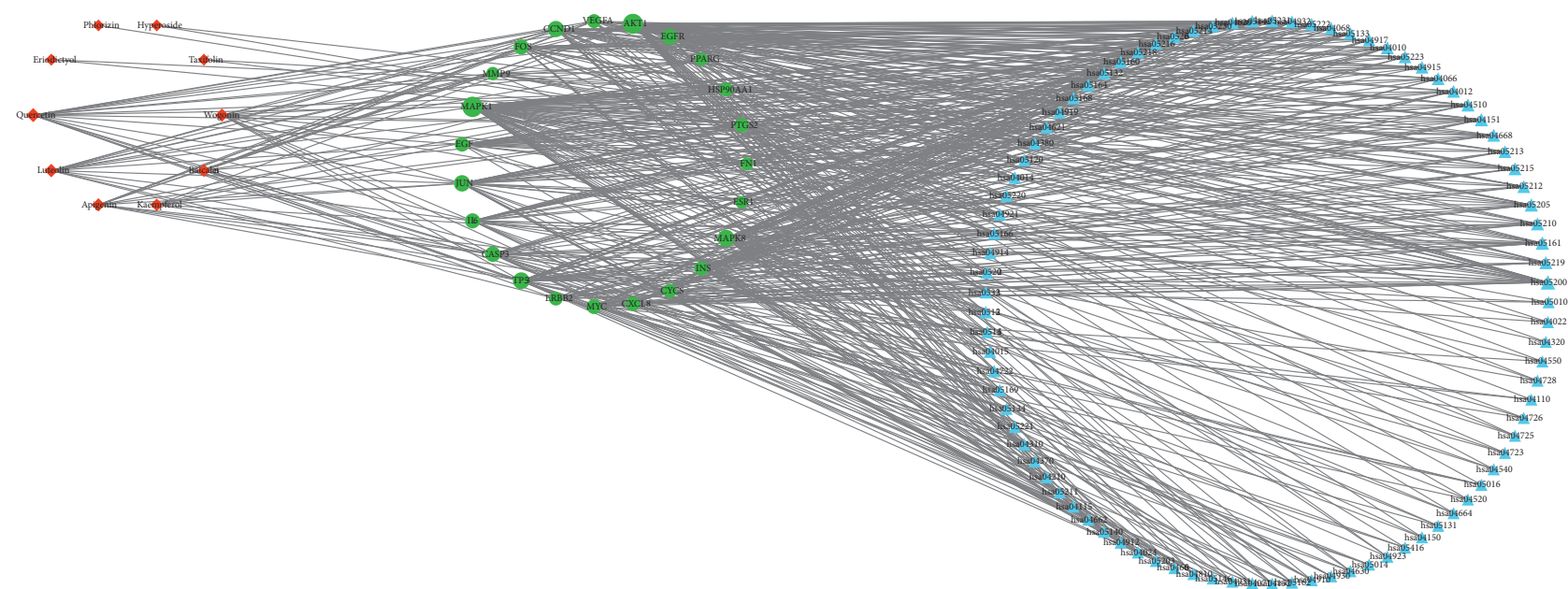

FIGURE 5: Chemical-target genes-pathway network: red represents chemical; blue represents target genes; green represents pathway.

\section{Conclusion}

In the present investigation, this finding revealed that P. freyniana possessed 43 flavonoids (40 of them was first reported) with 23 core target genes, which were associated with PI3K-Akt, MAPK, TNF signaling pathway, and pathway in cancer. This study demonstrated the multicompound, multitarget, and multimechanism of $P$. freyniana, which are very beneficial for the further study and utilization of this plant including the material basis and quality control research.

\section{Data Availability}

The data used to support the finding of this study are available from the corresponding author upon request.

\section{Conflicts of Interest}

The authors declare no conflicts of interest.

\section{Authors' Contributions}

Zaiqi Zhang and Wei Cai designed the study. Kailin Li, Wei Cai, Shihan Qin, Pei Xiong, Jie Peng, and Silin Shi performed the experiments. Kailin Li, Wei Cai and Shihan Qin performed data analyses. Kailin $\mathrm{Li}$ and Wei Cai wrote the experiments; Zaiqi Zhang, Shihan Qin, Pei Xiong, Jie Peng, and Silin Shi critically reviewed the manuscript. All authors read and approved the final manuscript. Wei Cai and Kailin Li contributed equally to this work.

\section{Acknowledgments}

This work was supported by the National Natural Science Foundation of China (no. 81603393), Natural Science Foundation of Hunan Province (no. 2018JJ3376), the Scientific Research Fund of Hunan Provincial Education Department (no. 19A353), Hunan Provincial Key Laboratory of Dong Medicine (no. 2015TP1020-03), and the platform construction project of Hunan Provincial key laboratory of Dong medicine (no. 2017CT5025).

\section{Supplementary Materials}

Figure S1: compounds-target genes. Figure S2: PPI network. Table S1: compounds-target genes. Table S2: GO analysis. Table S3: KEGG pathway. (Supplementary Materials)

\section{References}

[1] Editorial Board, Flora of China, Science Press, Beijing, China, 2003.

[2] Y. Zhong, X. Y. Qu, and Y. D. Zhang, "Medicinal Plants of potentilla genus in Xiangxi," Chinese Journal of Ethnomedicine and Ethnopharmacy, vol. 6, no. 72, pp. 344-345, 2014.

[3] K. M. Lu, Medicine of Dong Nationality, Guizhou Sciences Technology, Publishing House, Guizhou, China, 1992.

[4] Y. H. Cai, L. Liu, D. X. Han, and C. L. Liu, "Study on chemical components of potentilla freyniana bornm," Journal Wuhan University Technology, vol. 27, no. 9, pp. 32-33, 2014.

[5] X. H. Wu, C. Mi, Y. He et al., "Polyphenols from the rhizomes of potentilla freyniana," Medicinal Chemistry, vol. 5, pp. 21-22, 2015.

[6] X.-H. Wu, J.-L. Ruan, and Y.-L. Cai, "Triterpenes from the rhizomes of potentilla freyniana," Biochemical Systematics and Ecology, vol. 7, no. 4, pp. 509-511, 2009.

[7] H. Z. Chen, "Study on the chemical constituents of ethy acetate extract from Potentilla freyniana," Hubei University Chinese Medicine Dissertation, vol. 36, no. 5, pp. 744-746, 2015.

[8] S. H. Wang and G. D. Chen, "Studies on anti-inflammatory and immune effect Potentilla freyniana Bornm." Journal Hubei University Chinese Medicine, vol. 11, pp. 26-28, 2009.

[9] B. J. Xiao, G. D. Chen, Y. M. Zhu et al., "Study on anti-inflammatory and analgesic effect of Potentilla freyniana Bornm." Tianjin Medicine Journal, vol. 37, pp. 482-484, 2006.

[10] N. Satheeshkumar, S. Shantikumar, and M. Komali, "Identification and quantification of aldose reductase inhibitory flavonoids in herbal formulation and extract of gymnema sylvestre using HPLC-PDA and LC-MS/MS," Chromatography Research International, vol. 2014, Article ID 518175, 8 pages, 2014.

[11] Y. S. Fan, Y. M. Li, Y. Y. Wu et al., "Identification of the chemical constituents in simiao wan and rat plasma after oral administration by GC-MS and LC-MS," Evidence-Based Complementary 
and Alternative Medicine, vol. 2017, Article ID 6781593, 13 pages, 2017.

[12] L. Liu, J. Zhang, B. Zheng et al., "Rapid characterization of chlorogenic acids in duhaldea nervosa based on ultra-highperformance liquid chromatography-linear trap quadropoleorbitrap-mass spectrometry and mass spectral trees similarity filter technique," Journal of Separation Science, vol. 41, no. 8, pp. 1764-1774, 2018.

[13] M. Chandradevan, S. Simoh, A. Mediani et al., "UHPLC-ESIOrbitrap-MS analysis of biologically active extracts from gynura procumbens (lour.) merr. and Cleome gynandra L. Leaves," Evidence-Based Complementary and Alternative Medicine, vol. 2020, Article ID 3238561, 14 pages, 2020.

[14] X. D. Ye, Y. H. Wang, J. P. Zhao et al., "Identification and characterization of key chemical constituents in processed gastrodia elata using UHPLC-MS/MS and chemometric methods," Journal of Analytical Methods in Chemistry, vol. 2019, Article ID 4396201, 10 pages, 2019.

[15] Y. Chen, D. Chen, S. Liu et al., "Systematic elucidation of the mechanism of genistein against pulmonary hypertension via network pharmacology approach," International Journal of Molecular Sciences, vol. 20, no. 22, p. 5569, 2019.

[16] Y.-F. Zhang, Y. Huang, Y.-H. Ni, and Z.-M. Xu, "Systematic elucidation of the mechanism of geraniol via network pharmacology," Drug Design, Development and Therapy, vol. 13, pp. 1069-1075, 2019.

[17] J. Chen, J. Chen, and J. Lu, "Systematic elucidation of the mechanism of oroxylum indicum via network pharmacology," Evidence-Based Complementary and Alternative Medicine, vol. 2020, Article ID 5354215, 8 pages, 2020.

[18] W. Cai, K. L. Li, P. Xiong et al., "A systematic strategy for rapid identification of chlorogenic acids derivatives in duhaldea nervosa using UHPLC-Q-exactive orbitrap mass spectrometry," Arabian Journal of Chemistry, vol. 13, pp. 3751-3761, 2020.

[19] Y. J. Min, Y. P. Zhang, Q. Gao et al., "Study on the technology of extraction and content of flavonoids from potentilla chinensis series," Food and Fermentation Industries, vol. 6, pp. 176-179, 2008.

[20] M. A. Alvarez-Fernández, A. B. Cerezo, A. M. CañeteRodríguez et al., "Composition of nonanthocyanin polyphenols in alcoholic-fermented strawberry products using LC-MS (QTRAP), high-resolution MS (UHPLC-OrbitrapMS), LC-DAD, and antioxidant activity," Journal of Agricultural and Food Chemistry, vol. 63, pp. 2041-2051, 2015.

[21] C. Terreaux, Q. Wang, J. R. Ioset et al., "Complete LC/MS analysis of a Tinnevelli senna pod extract and subsequent isolation and identification of two new benzophenone glucosides," Planta Medica, vol. 68, pp. 349-354, 2002.

[22] A. Taamalli, D. Arráez-Román, L. Abaza et al., "LC-MS-based metabolite profiling of methanolic extracts from the medicinal and aromatic species mentha pulegium and origanum majorana," Phytochemical Analysis, vol. 26, pp. 320-330, 2015.

[23] M. Yasir, B. Sultana, and F. Anwar, "LC-ESI-MS/MS based characterization of phenolic components in fruits of two species of solanaceae," Journal of Food Science and Technology-Mysore, vol. 55, pp. 2370-2376, 2018.

[24] L. Bevilacqua, F. Buiarelli, F. Coccioli et al., "Identification of compounds in wine by HPLC-tandem mass spectrometry," Annali di Chimica, vol. 94, no. 9-10, pp. 679-690, 2004. 